\title{
ANALYTIC CONTINUATION OF RIEMANN'S ZETA FUNCTION AND VALUES AT NEGATIVE INTEGERS VIA EULER'S TRANSFORMATION OF SERIES
}

\author{
JONATHAN SONDOW
}

(Communicated by William W. Adams)

\begin{abstract}
We prove that a series derived using Euler's transformation provides the analytic continuation of $\zeta(s)$ for all complex $s \neq 1$. At negative integers the series becomes a finite sum whose value is given by an explicit formula for Bernoulli numbers.
\end{abstract}

\section{INTRODUCTION}

Euler computed the values of the zeta function at the negative integers using both Abel summation ( 75 years before Abel) and the Euler-Maclaurin sum formula. (Comparison of these values with those he found at the positive even integers led him to conjecture the functional equation 100 years before Riemann!) Euler also used a third method, his transformation of series or (E) summation (see $\S 2$ ), to calculate $\zeta(-n)$, but only for $n=0,1,2$, and 4 . (See $[1 ; 4, \S 1.5 ; 5$, volume 14 , pp. $442-443,594-595$; volume 15 , pp. 70-90; 7; 9, $\S \S 1.3,1.6,2.2,2.3 ; 10 ; 14$, Chapter III, $\S \S X V I I-X X]$.)

We observe in $\S 4$ that this last method in fact yields

$$
\zeta(-n)=(-1)^{n} B_{n+1} /(n+1) \text { for all } n \geq 0,
$$

but we require an explicit formula for Bernoulli numbers that was discovered a century after Euler. In $\S 3$ we justify the method by proving that a series used in $\S 4$ gives the analytic continuation of $\zeta(s)$ for all $s \neq 1$. Similar results for approximations to Euler's transformation are obtained in $\S 5$, as well as an evaluation of $\zeta^{\prime}(0) / \zeta(0)=\log 2 \pi$.

In a paper in preparation, the author will apply the method to other zeta functions and to Dirichlet $L$-series.

\section{EULER'S TRANSFORMATION OF SERIES}

Any convergent series of complex numbers, written with alternating signs as

$$
A=a_{1}-a_{2}+a_{3}-\cdots,
$$

Received by the editors December 8, 1991 and, in revised form, June 3, 1992; presented to the American Mathematical Society on January 10, 1992, at Baltimore, MD.

1991 Mathematics Subject Classification. Primary 11M06, 40G05, 11 B68. 
can also be written in the form

$$
A=\frac{1}{2} a_{1}+\frac{1}{2}\left[\left(a_{1}-a_{2}\right)-\left(a_{2}-a_{3}\right)+\cdots\right] .
$$

Repeating the process on the series in brackets, we have

$$
A=\frac{1}{2} a_{1}+\frac{1}{4}\left(a_{1}-a_{2}\right)+\frac{1}{4}\left[\left(a_{1}-2 a_{2}+a_{3}\right)-\left(a_{2}-2 a_{3}+a_{4}\right)+\cdots\right]
$$

and in general

$$
\sum_{1}^{\infty}(-1)^{n-1} a_{n}=\sum_{0}^{k-1} \frac{\Delta^{j} a_{1}}{2^{j+1}}+\sum_{n=1}^{\infty}(-1)^{n-1} \frac{\Delta^{k} a_{n}}{2^{k}}
$$

where $\Delta^{0} a_{n}=a_{n}$ and

$$
\Delta^{k} a_{n}=\Delta^{k-1} a_{n}-\Delta^{k-1} a_{n+1}=\sum_{m=0}^{k}(-1)^{m}\left(\begin{array}{l}
k \\
m
\end{array}\right) a_{n+m}
$$

for $k \geq 1$. It is proved in [11, §33B] that the sum of the last series in (1) approaches 0 as $k \rightarrow \infty$, so that

$$
\sum_{1}^{\infty}(-1)^{n-1} a_{n}=\sum_{0}^{\infty} \frac{\Delta^{j} a_{1}}{2^{j+1}}
$$

which is Euler's transformation of series. (See [5, volume 10, pp. 222-227; 9, $\S 4.6 ; 11, \S \S 35 \mathrm{~B}, 59,63]$.)

\section{AnAlytic CONTINUATION of $\zeta(s)$}

Instead of working directly with $\zeta(s)$, which for $\sigma=\operatorname{Re}(s)>1$ is given by $\zeta(s)=1^{-s}+2^{-s}+3^{-s}+\cdots$, let us consider the alternating series

$$
\zeta(s)-2 \cdot 2^{-s} \zeta(s)=1^{-s}-2^{-s}+3^{-s}-\cdots,
$$

which converges for $\sigma>0$ (see $\S 5$ ). Applying the Euler transformation, we have, for $\sigma>1$,

$$
\begin{aligned}
\left(1-2^{1-s}\right) \zeta(s) & =\sum_{0}^{\infty} \frac{\Delta^{j} 1^{-s}}{2^{j+1}} \\
& =\sum_{0}^{\infty} \frac{1-\left(\begin{array}{l}
j \\
1
\end{array}\right) 2^{-s}+\left(\begin{array}{l}
j \\
2
\end{array}\right) 3^{-s}-\cdots+(-1)^{j}\left(\begin{array}{l}
j \\
j
\end{array}\right)(j+1)^{-s}}{2^{j+1}} .
\end{aligned}
$$

Theorem. The analytic continuation of $\zeta(s)$ for all complex $s \neq 1$ is given by the product

$$
\zeta(s)=\left(1-2^{1-s}\right)^{-1} \sum_{0}^{\infty} \frac{\Delta^{j} 1^{-s}}{2^{j+1}}
$$

in which the series converges absolutely and uniformly on compact sets to an entire function.

Proof. Fix $k \geq 0$. Evidently

$$
\Delta^{k} n^{-s}=(s)_{k} \int_{0}^{1} \cdots \int_{0}^{1}\left(n+x_{1}+\cdots+x_{k}\right)^{-s-k} d x_{1} \cdots d x_{k}
$$


for $k=1,2, \ldots$, where $(s)_{k}$ denotes the product $s(s+1) \cdots(s+k-1)$. Hence,

$$
\left|\Delta^{k} n^{-s}\right| \leq\left|(s)_{k}\right| / n^{\sigma+k} \quad \text { whenever } \sigma+k \geq 0,
$$

$k=0,1,2, \ldots$, where $(s)_{0}=1$. Now let $S$ be a compact set in the half plane $\sigma>1-k$, and let $M_{n}$ denote the maximum of $\left|(s)_{k}\right| / n^{\sigma+k}$ on $S$. Then (6) implies that $\sum M_{n}$ dominates the series

$$
\sum_{n=1}^{\infty}(-1)^{n-1} \Delta^{k} n^{-s}
$$

on $S$. It follows, using the triangle inequality, that the Euler transform of $\sum M_{n}$ dominates the Euler transform of (7), which, since $\Delta^{j} \Delta^{k}=\Delta^{j+k}$, is

$$
\sum_{j=0}^{\infty} \frac{\Delta^{j} \Delta^{k} 1^{-s}}{2^{j+1}}=\sum_{j=k}^{\infty} \frac{\Delta^{j} 1^{-s}}{2^{j+1-k}}
$$

Multiplying this by $1 / 2^{k}$ and adding $\sum_{0}^{k-1} \Delta^{j} 1^{-s} / 2^{j+1}$ produces the series in (4), which, since $k$ is arbitrary, therefore converges absolutely and uniformly on compact sets to an entire function. Since the series in (3) has zeros at the (simple) poles of $\left(1-2^{1-s}\right)^{-1}$ except at $s=1$ (for a direct proof see [12]), the theorem follows.

\section{Evaluation of $\zeta(-m)$}

Let $m$ be a positive integer or 0 . Note that $(-m)_{j}=0$ and, hence, $\Delta^{j} 1^{m}=$ 0 for $j>m$. Thus when $s=-m$ the series in (5) becomes a finite sum. Its value is given by a formula for Bernoulli numbers that Carlitz [2] attributes to Worpitzky [15] (see also [3]), namely, the second equality in the following.

Corollary. For $m=0,1,2, \ldots$,

$$
\zeta(-m)=\frac{1}{1-2^{m+1}} \sum_{0}^{m} \frac{\Delta^{j} 1^{m}}{2^{j+1}}=(-1)^{m} \frac{B_{m+1}}{m+1} .
$$

Alternatively, one can view $\zeta(-m)=(-1)^{m} B_{m+1} /(m+1)$ as known, which gives a proof of Worpitzky's formula (compare [6]).

\section{APPROXIMATIONS TO EULER'S TRANSFORMATION}

Note that (1), (3), and (6) imply (without using (2)) that for $k \geq 1$ the product

$$
\zeta(s)=\left(1-2^{1-s}\right)^{-1}\left(\sum_{0}^{k-1} \frac{\Delta^{j} 1^{-s}}{2^{j+1}}+\frac{1}{2^{k}} \sum_{n=1}^{\infty}(-1)^{n-1} \Delta^{k} n^{-s}\right)
$$

provides the analytic continuation of $\zeta(s)$ on the punctured half plane $\sigma>1-k$, $s \neq 1$ where the infinite series converges absolutely and uniformly on compact sets to a holomorphic function. Moreover, except that the convergence will not be absolute in the strip $-k<\sigma \leq 1-k$, this remains true for $k \geq 0$ and $\sigma>-k$, 
$s \neq 1$. (Proof. Grouping terms in pairs in the even partial sums of the second summation, we have

$$
\begin{aligned}
\sum_{n=1}^{2 N}(-1)^{n-1} \Delta^{k} n^{-s} & =\sum_{n=1}^{N}\left(\Delta^{k}(2 n-1)^{-s}-\Delta^{k}(2 n)^{-s}\right) \\
& =\sum_{n=1}^{N} \Delta^{k+1}(2 n-1)^{-s} .
\end{aligned}
$$

Then it follows from (6) that both even and odd partial sums converge as required.) Since we can use (8) with $k \geq m+1$ to evaluate $\zeta(-m)$, the approximations (1) to Euler's transformation yield everything it does except formula (5).

As an example, take $k=1$ in (8):

$$
\zeta(s)=\left(1-2^{1-s}\right)^{-1}\left(\frac{1}{2}+\frac{1}{2} \sum_{1}^{\infty}(-1)^{n-1}\left(n^{-s}-(n+1)^{-s}\right)\right)
$$

for $\sigma>-1, s \neq 1$. (This formula appears in Hardy's proof of the functional equation $[8 ; 13, \S 2.2]$ and gave the idea for the present note.) Thus $\zeta(0)=-1 / 2$ and, using Wallis's product for $\pi / 2$,

$$
\frac{\zeta^{\prime}(0)}{\zeta(0)}=2 \log 2+\log \frac{2}{1} \frac{2}{3} \frac{4}{3} \frac{4}{5} \cdots=\log 2 \pi,
$$

which figures in the Hadamard product representation of the zeta function.

\section{REFERENCES}

1. R. Ayoub, Euler and the zeta function, Amer. Math. Monthly 81 (1974), 1067-1086.

2. L. Carlitz, Remark on a formula for the Bernoulli numbers, Proc. Amer. Math. Soc. 4 (1953), 400-401.

3. E. Cesàro, Transformations algébriques par le calcul des différences, Nouv. Ann. Math. (3) 5 (1886), 489-492.

4. H. M. Edwards, Riemann's zeta function, Academic Press, New York, 1974.

5. L. Euler, Opera Omnia, Series Prima, Teubner, Leipzig and Zurich, 1911-1956.

6. H. L. Garabedian, A new formula for the Bernoulli numbers, Bull. Amer. Math. Soc. $\mathbf{4 6}$ (1940), 531-533.

7. D. Goss, $A$ simple approach to the analytic continuation and values at negative integers for Riemann's zeta function, Proc. Amer. Math. Soc. 81 (1981), 513-517.

8. G. H. Hardy, A new proof of the functional equation for the zeta-function, Mat. Tidsskrift B 1922, 71-73.

9. __ Divergent series, Oxford Univ. Press, London, 1949.

10. M. Kline, Euler and infinite series, Math. Mag. 56 (1983),307-315.

11. K. Knopp, Theory and application of infinite series, Blackie \& Sons, London, 1951.

12. J. Sondow, The zeros of the alternating zeta function on $\sigma=1$, preprint.

13. E. C. Titchmarsh, The theory of the Riemann zeta-function, Oxford Univ. Press, London, 1951.

14. A. Weil, Number theory, an approach through history, Birkhäuser, Basel, 1984.

15. J. Worpitzky, Studien über die Bernoullischen und Eulerschen Zahlen, J. Reine Angew. Math. 94 (1883), 203-232. 